\title{
Conflictos raciales a la luz de la teoría de los juegos
}

\author{
Carlota Solé \\ Universitat Autònoma de Barcelona. Facultat de Ciències Polítiques \\ Departament de Sociologia. 08193 Bellaterra (Barcelona). Spain
}

\section{Resumen}

El artículo trata de los conflictos raciales derivados de la discriminación ante el mercado de trabajo de los inmigrantes procedentes del Tercer Mundo residentes en un distrito compuesto por cuatro barrios de la ciudad de Barcelona, donde la concentración de la inmigración interior y exterior es muy alta en comparación con otros barrios de la misma ciudad y otros municipios españoles. Se analizan las actitudes y la conducta de los agentes sociales, a partir de los datos de dos encuestas complementarias sobre las condiciones de vida y de trabajo de los colectivos de inmigrantes extranjeros y sobre las acritudes reacias, xenófobas de la población autóctona en relación a la presencia de extranjeros. Se diseñan las distintas estrategias y preferencias para cada uno de los agentes sociales o jugadores: Administración, empresarios, sindicatos, trabajadores autóctonos y trabajadores extranjeros. Se plasman en matrices de juego los conflictos potenciales entre unos y otros, por la confluencia antagónica o no de los distintos intereses en juego. De esta forma se visualizan las situaciones de conflicto y su posible resolución.

Palabras clave: inmigración extranjera, racismo, conflicto racial, teoría de los juegos.

\begin{abstract}
Racial conflicts and game theory analysis
This article deals with racial conflicts due to market discrimination of Third World immigrants in a Barcelona's district where the concentration of internal and external immigration is high compared with other districts and other Spanish towns and villages. According to survey data on labour and life conditions of immigrants, on the one hand; and xenophobic and racist attitudes, on the other, the strategies and preferences of the diverse social agents o players (Administration, employers, trade unions, Spanish workers, foreign workers) are drawn. Game theory is applied and conflict is shown through the matrixes which present different solutions of a conflict situacion.
\end{abstract}

Key words: foreign immigration, racism, racial conflict, game theory.

\section{Sumario}

1. Situaciones de conflicto 3. Bibliografía

2. Análísis estratégico 


\section{Situaciones de conflicto}

Los omnipresentes factores estructurales de la pobreza de muchos países del llamado Tercer Mundo, han dado lugar a que en estos últimos seis a diez af́os hayan afluido a Cataluña y, en concreto, a Barcelona, un número no despreciable de marroquíes, argelinos, gambianos, senegaleses, paquistanies, filipinos, coreanos y otros extranjeros. Aunque de distinta suerte en su trayectoria migratoria y asentamiento en la ciudad, gran parte de estas personas se encuentran con enormes dificultades para abrirse camino en su nueva ubicación territorial y social.

En una ciudad en que las posibilidades de empleo y salarios al alza se han hecho realidad en algunos sectores económicos y en otros, la reestructuración constante ha asegurado un crecimiento notable en el nivel de ocupación con motivo de la celebración de los Juegos Olímpicos en 1992; los colectivos de marroquíes, argelinos, gambianos, senegaleses, paquistaníes, filipinos, etc., han afrontado una situación de clara discriminación ante el mercado de trabajo, de abuso en encontrar una vivienda donde instalarse y de rechazo en su convivencia con la población autóctona. En este escrito presentamos algunas reflexiones sobre la situación de conflicto latente que deriva de la irrupción de estos colectivos en el mercado de trabajo controlado por los empresarios y sindicatos autóctonos, stuación de conflicto que se puede modelar y visualizar con mayor precisión aplicando la teoría de los juegos a los datos empíricos de tres surveys.

La situación de conflicto que deriva de la discriminación de los inmigrantes extranjeros ante el mercado de trabajo y de las actitudes reacias, xenofobas, de la población autóctona que considera lesionados sus derechos a conseguir la totalidad de los puestos de trabajo y de los beneficios de los servicios sociales de las instituciones españolas, y no acepta con agrado que estos colectivos puedan asociarse para defender sus intereses; puede plasmarse en las matrices de los distintos juegos o situaciones en las que se relacionan los distintos actores sociales o jugadores (inmigrantes extranjeros, trabajadores españoles, empresarios, sindicatos) que siguen una conducta o estrategia (serie de decisiones racionales ordenadas de acuerdo con las propias preferencias), teniendo en cuenta que el otro actor social o jugador contrincante es tan racional como uno mismo y tendrá igualmente diseñado su orden de preferencias. Así, puede ofrecerse una explicación, si no alternativa, tal vez complementaria y, por supuesto, más plástica, a la descripción que proporcionan las encuestas como técnica sociológica cuantitativa más solvente, por el momento. Aplicando la teoría de los juegos, pueden clarificarse estrategias o relaciones entre los inmigrantes extranjeros y los demás actores sociales que, de otra forma, permanecerían encubiertas o menos explícitas.

1. Solé, C. Encuesta de 1987 a los inmigrantes del Tercer Mundo, publicada por el Centro de Investigaciones Sociológicas de Madrid en 1991.

- Encuesta de 1990 sobre racismo en Espana, CES, Madrid (en prensa). 
A este efecto es necesario definir las actuaciones de los actores sociales en situaciones conflictivas en el mercado de trabajo que derivarán en discriminación de los grupos sociales o colectivos minotitarios en relación a otros mayoritarios. Desde la perspectiva sociológica la discriminación se valora en sus distintos grados según la distancia entre los diversos niveles de renta o ingresos, status social, prestigio, poder en la escala o estructura social. Si introducimos en esta perspectiva el enfoque económico (G. Becker, 1976), para explicar la discriminación en el mercado de trabajo debe completarse el análisis sociológico de las causas de la discriminación con el análisis de las consecuencias económicas de este hecho. A tal efecto es preciso introducir una magnitud monetaria, el dinero, como indicador objetivo para medir la discriminación. Si un individuo tiene una tendencia o inclinación hacia la discriminación, es decir, tiende a preferir un grupo a otro; tiene que actuar como si quisiera pagar algo directamente o en forma de renta o ingresos más reducidos, para que le asocien con unas personas y no con otras. Cuando se produce la verdadera discriminación debe, en realidad, pagar por este privilegio. Así se llega al núcleo del prejuicio y la discriminación.

La discriminación de un individuo o grupo contra otro u otros puede ser consciente y voluntaria o inconsciente e involuntaria. En el primer caso, a la distancia social entre uno y otro en términos de relativo status socioeconómico se añade la substituibilidad del segundo en el proceso productivo. Otro factor a tener en cuenta es la dimensión del grupo discriminado. El aumento numérico del grupo minoritario puede incrementar los prejuicios contra él por parte del grupo mayoritario, que teme un crecimiento relativo de su competencia y poder en el ámbito en que coexisten; o, por el contrario, puede conllevar un mayor contacto y conocimiento mutuos que induce a paliar los prejuicios existentes contra el grupo minoritario. En el segundo caso la ignorancia sobre las características del grupo minoritario (nivel educativo de los africanos occidentales, formación profesional de las filipinas que emigran a España, por ejemplo, que les capacitan para llevar a cabo otras tareas que las labores de recolección de fruta o el servicio doméstico que realizan en nuestro país) favorables a los intereses del grupo mayoritario discriminador conduce a situaciones de discriminación. El mayor contacto y conocimiento mutuo puede eliminar esta forma involuntaria de discriminación.

Estos factores: prejuicios, ignorancia, además de la localización geográffca (zonas rurales o urbanas, países desarrollados o subdesarrollados) y cronológica, amén de la estructura de la personalidad de cada individuo, dan lugar a una inclinación o tendencia hacia la discriminación presente entre empresarios, trabajadores, consumidores, sindicatos, etc. que puede objetivarse, siguiendo a G. Becker (1971), a través del coeficiente individual de discriminación.

Por otro lado, dado un mercado de trabajo dual (Ch. Sabel, 1985): por un lado, casi totalmente transparente para los trabajadores autóctonos, arraigados en el pais, especializados, con acceso a la información, know how, y por otro lado, casi totalmente opaco para otro grupo de trabajadores inmigrantes, no 
especializados, con casi nula información sobre las condiciones de trabajo, salarios, acción sindical en la sociedad receptora; existe competencia entre los trabajadores autóctonos e inmigrantes que en los años 1960 llegaron a Cataluña desde el sur de España (inmigrantes interiores) y los trabajadores procedentes de los países del denominado Tercer Mundo, Ilegados a Cataluña a finales de los años setenta y la década de los ochenta (inmigrantes extranjeros). Los trabajadores inmigrantes extranjeros juegan un papel de ejército de reserva respecto al resto de los trabajadores españoles. La diferencia más notable entre ambos colectivos, actores sociales o jugadores, es que los primeros deben legalizar su situación como residentes y trabajadores en España, mientras que los segundos son ciudadanos del Estado español.

La progresiva especialización de las actividades productivas da lugar a una segmentación muy fuerte del mercado de trabajo que puede llegar a correlacionarse estrechamente con los grupos étnico-culturales. Así, las tareas más sucias, rutinarias, repetitivas, etc., que corresponden a ocupaciones que se sitúan en los estratos más bajos de la estructura ocupacional, son rechazadas por los trabajadores autóctonos y, también progresivamente, en la medida en que experimentan movilidad ocupacional, por los trabajadores inmigrantes interiores. Estas tareas son cubiertas por los inmigrantes extranjeros. Otras actividades que exceden a la mano de obra disponible son también realizadas por los extranjeros inmigrados de los países del Tercer Mundo. Ahora bien, existe una franja de actividades y tareas realizadas por trabajadores semi-especializados o poco cualificados, generalmente inmigrantes interiores, que temen la competencia de los inmigrantes procedentes del Tercer Mundo. Cabe reconocer que existen tres situaciones en el mercado de trabajo que pueden conducir a la discriminación: sustituibilidad de la mano de obra, complementariedad y competencia.

Los jugadores son:

- La Administración que regula la entrada de extranjeros en el país, acuerda su legalización y la regularización de su residencia y trabajo.

- Los empresarios que dan trabajo, a ser posible reduciendo al máximo los costes de producción o distribución, entre otros los costes salariales.

- Los sindicatos que, en la España actual se definen como sindicatos de clase e intentan representar los intereses del conjunto de la clase trabajadora, englobando a los trabajadores legales como a los ilegales, a la vez que reivindican anualmente mejores condiciones de trabajo y aumentos salariales para los trabajadores (legales) afiliados a ellos.

- Los trabajadores autoctonos e inmigrantes desde hace tiempo en Cataluña que han alcanzado un nivel elevado de especialización en su trabajo, han tenido movilidad ocupacional a lo largo de su trayectoria profesional, han luchado muchos años por conseguir unos salarios elevados y condiciones de trabajo dignas, y los trabajadores inmigrantes interiores y los autóctonos que no han alcanzado un nivel de especialización suficientemente elevado para competir por trabajos cualificados, de alta remuneración, han experimentado una escasa movilidad ocupacional en su trayectoria laboral y han lucha- 
do por salir de situaciones de discriminación respecto al sector de los trabajadores cualificados.

- Los trabajadores inmigrantes extranjeros procedentes del Tercer Mundo dispuestos a conseguir un puesto de trabajo a cualquier precio, a pesar de que no se corresponda a su nivel de formación elevado en relación a los trabajos manuales, sucios y rutinarios que encuentran con relativa mayor facilidad en el mercado de trabajo.

\section{Análisis estratégico}

Al aplicar la teoría de los juegos, como derivada del análisis de la elección racional, a las situaciones de conflicto entre empresarios, trabajadores españoles y extranjeros y sindicatos que tratamos de modelar para conocer si existe una solución al conflicto de intereses no previsible a partir del análisis estricto de los datos empíricos de la Encuesta sobre Racismo de 1990, las preferencias de los actores sociales mencionados, o jugadores, y su orden, responden a la realidad de un espacio y tiempos determinados. Al determinar las preferencias de los jugadores intervinientes y su orden, se han tenido en cuenta las condiciones sociales y/o institucionales que hacen posible o imposible la formación y diseno de unas y otras preferencias.

De esta forma, las preferencias de los actores sociales están contextualizadas dentro de los límites de los recursos disponibles para los jugadores o actores, su capacidad de aprendizaje, sus prioridades y los resultados de modos alternativos de comportamiento estratégico. Sin embargo, esta contextualización es ahistórica por cuanto, a lo sumo, se refiere al espacio temporal de la realización de una encuesta o de la observación empírica de unas actitudes u opiniones. Por otro lado, la formalización en matrices de juego pretende no sólo presentar de forma más plástica las siruaciones de conflicto una vez modeladas, sino también descubrir relaciones conflictivas entre los agentes sociales, no aparentes o encubiertas en el análisis histórico-descriptivo de los datos de una encuesta. Así, aunque no pueda decirse que el análisis de la elección racional o rational choice sea aplicable a todos y cada uno de los temas de nutestra disciplina, algunos aspectos de un problema sociológico pueden ser tal vez más claramente explicitados e incluso explicados bajo el presupuesto y asunción de la racionalidad estratégica. La lógica de una situación es modelizada sobre la base de la interdependencia de las decisiones y preferencias de un individuo con respecto a las decisiones y preferencias de los demás individuos.

Akerlof (1984) ofrece una visión alternativa a la de Becker para explicar el empleo precario de trabajadores inmigrantes y otras minorías sociológicas, a partir del "principio de las mercaderías de oportunidad o cacharros" (Lemons Principle). Ante la perspectiva de poder emplear a trabajadores autóctonos (grupo mayoritario) o inmigrantes extranjeros (grupo minoritario), los empresarios tenderán a ocupar mano de obra extranjera, desplazando a la autóctona, dada la asimétrica información que existe en el mercado de trabajo para todos y cada uno de los jugadores respecto a los demás actores en liza. 
En estas condiciones la estrategia de los empresarios es la de rebajar al máximo los costes salariales por trabajo especializado realizado con calidad suficiente para ser rentable. A los empresarios les interesará que persista la situación de ilegalidad de una parte de mano de obra para poder pagar salarios bajos. Los trabajadores extranjeros en España tienen, en muchos casos, cualificaciones algo superiores a las correspondientes a los empleos que consiguen en España. Aunque la regularización de los extranjeros depende de la Administración y no de los empresarios, éstos calcularán racionalmente sus expectativas de beneficio areniéndose al control social por parte de los sindicaros para que se pague el salario interprofesional a los trabajadores legales (españoles y extranjeros regularizados) por las ocupaciones en el mercado formal de trabajo. Estas ocupaciones llevan adscriras generalmente acrividades especializadas que requieren mano de obra especializada que puede ser extranjera. En el segmento informal del mercado de trabajo no existe un control social férreo sobre los empresarios, sobre los salarios y condiciones de trabajo que cobran y tienen los obreros, sean españoles o extranjeros.

La estrategia de los inmigrantes extranjeros es la de entrar en el mercado de trabajo a cualquier precio, intentando mejorar su situación. Se presupone que para los inmigrantes extranjeros es prioritario ganarse la vida a ser legales, aunque muchos intentarán situarse en el segmento del mercado de trabajo formal para alcanzar niveles de vida superiores, equiparables a los de la población activa española. Se presupone que los empresarios intentarán minimizar los costes laborales que ellos consideran de enorme peso en los costes globales.

Las estrategias de los empresarios serán:

a) tener a mano un colectivo de trabajadores inmigrantes, muchos de los cuales son cualificados y capaces de realizar tareas especializadas, a los que contratar por bajos salarios, lo cual implica la no legalización de los extranjeros; o bien,

b) no poder evitar la legalización de los trabajadores procedentes del Tercer Mundo, contratar y pagar los mismos salarios a españoles y extranjeros.

Por su parte, los trabajadores extranjeros adoptarán las siguientes estrategias:

a) entrar en el mercado formal de trabajo, cobrando igual salario que los trabajadores autóctonos; o bien,

b) percibir salarios inferiores por igual trabajo.

Las preferencias de los empresarios son:

- Valor 4: que las tareas especiadizadas las hagan los trabajadores extranjeros preferentemente, excluyendo a los españoles, por salarios inferiores al interprofesional ( $\mathrm{P}_{\mathrm{B}}$ : pagar salarios bajos).

- Valor 3: que las tareas especializadas las puedan realizar santo los trabajadores españoles como los extranjeros, por el mismo salario ( $P_{\text {ig: }}$ pagar salarios iguales). 
- Valor 2: que las tareas especializadas las realicen los espantoles y que las actividades no especializadas las lleven a cabo los trabajadores extranjeros, por salarios diferentes, inferiores en el segundo caso al interprofesional $\left(\mathrm{P}_{\mathrm{B}}\right.$ : pagar salarios bajos).

- Valor 1: que las tareas no cualificadas y no especializadas las realicen tanto los trabajadores españoles como los extranjeros por el salario interprofesional ( $P_{\mathrm{ig}}$ : pagar salarios iguales).

Las preferencias de los inmigrantes extranjeros son:

- Valor 4: trabajar en tareas cualificadas del mercado de trabajo formal a igual salario que los trabajadores españoles ( $S_{\text {ig: }}$ salarios iguales).

- Valor 3: trabajar en tareas no cualificadas por un salario igual que el de los trabajadores españoles ( $\mathrm{S}_{\text {ig: }}$ : salarios iguales).

- Valor 2: trabajar en tareas no cualificadas en el mercado informal por un salario inferior al que perciben los trabajadores españoles $\left(S_{\text {inf: salarios infe- }}\right.$ riores).

- Valor 1: trabajar en tareas cualificadas o especializadas por un salario inferior al que cobran los trabajadores españoles, aun habiendo regularizado su situación $\left(\mathrm{S}_{\text {inf }}\right.$ salarios inferiores $)$.

El orden de preferencias es:

Empresarios:

Trabajadores extranjeros:

Valor 4 Valor 3 Valor 2 Valor 1

\begin{tabular}{llll}
\hline $\mathrm{P}_{\mathrm{B}}, \mathrm{S}_{\text {inf }}$ & $\mathrm{P}_{\mathrm{ig}}, \mathrm{S}_{\mathrm{ig}}$ & $\mathrm{P}_{\mathrm{ig}}, \mathrm{S}_{\text {inf }}$ & $\mathrm{P}_{\mathrm{B}}, \mathrm{S}_{\mathrm{ig}}$ \\
$\mathrm{P}_{\mathrm{ig}}, \mathrm{S}_{\mathrm{ig}}$ & $\mathrm{P}_{\mathrm{B}}, \mathrm{S}_{\mathrm{ig}}$ & $\mathrm{P}_{\mathrm{B}}, \mathrm{S}_{\mathrm{inf}}$ & $\mathrm{P}_{\mathrm{ig}}, \mathrm{S}_{\text {inf }}$
\end{tabular}

La estructura de las decisiones se plasma en la matriz siguiente:

Matriz I

Tr. ext.

\begin{tabular}{|c|c|c|}
\hline & $S_{i g}$ & $S_{\text {inf }}$ \\
\hline$N L-P_{B}$ & 1,3 & 4,2 \\
\hline $\mathrm{L}-\mathrm{P}_{\mathrm{ig}}$ & 3,4 & 2,1 \\
\hline
\end{tabular}

Los inmigrantes tienen una estrategia dominante: intentar entrar en el mercado de trabajo a cualquier precio cobrando igual salario que los españoles. El resultado es $(3,4)$ un resultado de equilibrio estable: los empresarios presionarán para que persista la posibilidad de poder contratar mano de obra por salarios inferiores al interprofesional $y$, por lo tanto, harán lo posible para que no se regularice la situación de los inmigrantes extranjeros y éstos presionarán 
para poder legalizar su situación y poder competir en el mercado de trabajo en igualdad de condiciones con los espanioles.

Este es el máximo resultado posible, aunque los empresarios preferirían seguir contratando mano de obra ilegal por salarios bajos (casilla superior derecha, donde alcanzan el valor 4), pero en esta situación los inmigrantes presionarían, a través de los sindicatos y la Administración, para cobrar salarios iguales a los que perciben los españoles y para regularizar su situación definitivamente. El papel de la Administración ante esta eventualidad es, por supuesto, mtry importante, al decidir sobre la legalización o no de los extranjeros. Dadas las dificultades de regularizar su situación, en realidad siempre habrá inmigrantes dispuestos a trabajar en tareas inferiores a las que corresponden a su nivel de formación. Es decir, temporalmente o coyunturalmente, siempre habrá trabajadores que acepten cualquier empleo por un salario inferior al interprofesional. En este caso, la solución del juego sería $(4,2)$, según la cual los empresarios conseguirían mano de obra a bajo precio y los inmigrantes alcanzarían a colocarse en el mercado de trabajo y ganarse la vida minimamente, aunque fuera dejando en las manos de los obreros españoles la prioridad de optar por las tareas especializadas. Este resultado se acerca más a la realidad empírica por cuanto, a pesar de tener una estrategia determinante (cobrar igual salario que los españoles), los extranjeros cederán ante la presión de los empresarios en abaratar costes y ante la falta de reacción de los sindicatos en la defensa de sus intereses como trabajadores inmigrantes.

La matriz diseñada muestra una situación de conflicto frecuente entre empresarios y trabajadores extranjeros en la España actual. Los extranjeros son minoría, numérica y sociológicamente; se comportan racionalmente priorizando la supervivencia en nuestro país. Igualmente racionales son los empresarios y obreros españoles desde sus respectivas opticas. El análisis estratégico presenta de forma plástica situaciones de conflicto descritas empiricamente a través de los datos de los surveys. Sin entrar en la discusión polémica sobre el mayor o menor contenido heurístico de la aproximación del análisis racional sobre la acción colectiva en comparación con el estrictamente empírico de las encuestas, la teoría de los juegos aporta nueva luz sobre un nuevo tipo de conflicto: el racial, presumiblemente más frecuente y crudo en los próximos años en Europa.

\section{Bibliografia}

AKERLOF, G.A. (1986). An Economic Theorist's Book of Tales. Cambridge: CUP, 1984. BECKER, G.A. (1971). The Economics of Discrimination. Chicago, London: Chicago University Press.

- (1976). The Economic Approach to Human Behaviour. Chicago, London: Chicago University Press.

SABEL, Ch. (1985). Trabajo y Poltitica. Madrid: Ministerio de Trabajo.

SOlE, C. y HeRrerA, E. (1991). Los trabajadores extranjeros en Cataluña. Madrid: CIS.

SOLE, C. Racismo y discriminación en el mercado de trabajo. Madrid: CES (en prensa). 